\title{
Materials' physics and chemistry for space application
}

\author{
Ugo Lafont ${ }^{1} \cdot$ Adrian Tighe $^{1}$
}

Published online: 29 June 2021

(c) CEAS 2021

It is a great pleasure for us to present this special issue of the CEAS Space Journal devoted to Materials' Physics and Chemistry for Space Application. This issue compiles recent results and reviews on the development of materials for space application as well as the effect of the space environment on materials' behavior. The issue is dedicated to the memory of our former colleague Christopher Semprimoschnig, to whom we have also dedicated the first contribution.

The development of new materials with enhanced functionality is one of the cornerstones of technological breakthrough for demanding applications like space. Whether driven by more stringent mission requirements, or originating from non-space application, materials with optimized, enhanced or new functionalities are a strong enabler for the conception of any new space mission. However, to take full advantage of the properties from the available materials developed, it is of upmost importance to understand how their functionality is affected by the space environment. In short, understanding materials' physics and chemistry for space application is a prerequisite to designing a safe and successful mission.

The first part of this special issue will be related to new materials development and material combination in the field of energy conversion, structural monitoring, self-healing concepts, materials aging and processing. The second part will focus on the efforts made worldwide to assess material behavior and reliability in the space environment, highlighting the specific ground-based experiments designed for this purpose.

The first paper by Candolfi et al. presents an overview of thermoelectric materials development for space applications

Ugo Lafont

ugo.lafont@esa.int

Adrian Tighe

adrian.tighe@esa.int

1 European Space Research and Technology Centre-

European Space Agency, Keplerlaan 1, PO Box 299,

2200 AG Noordwijk, The Netherlands for radioisotope thermoelectric generators. Several thermoelectric materials families are presented that show promising performances.

The second contribution by Pernigoni et al. reviews the ongoing research on the development of space compliant self-healing concepts and associated materials to improve damage tolerance of specific systems, with ballistic impact protection as the main application.

In the field of materials for space, carbon fiber-reinforced polymers (CFRP) are extensively used. The paper by Rocha et al. is related to the implementation of embedded sensing capability that is used for manufacturing process monitoring and residual strain evaluation. The following paper from Quinn et al. deals with the assessment of the effect of vacuum on additive manufacturing processes using thermoplastics. The results highlight the potential for the development of in-space/out-of-earth manufacturing capabilities.

Before being exposed to the space environment, some materials can degrade on ground. The paper by Wolfberger et al. shows how long-term storage can affect the chemical nature of greases and lubricants.

Moving towards the low earth orbit environment, the effect of atomic oxygen (ATOX) on materials degradation needs to be taken into account. It is know that polyimide film can suffer from ATOX erosion; the contribution from Tagawa et al. shows how the presence of nitrogen affects the erosion in a simulated sub low earth orbit environment. On the other hand, the contribution from Wright shows how materials can be enhanced to increase their ATOX erosion resistance using organic/inorganic nanocomposites. The following paper from Goto et al. presents data from the MDM2 flight experiment, assessing the effect of ATOX on protective materials.

The contribution from Kleiman et al. is related to the geostationary earth orbit environment. To improve their resistance to the space environment, the materials are treated using an ion-beam deposition process. An overview of results achieved throughout the years is presented, showing that the treated materials have enhanced radiation stability 
and can be produced with controlled surface resistivity over a wide range of charge dissipation values.

The recent scientific missions from ESA are very challenging in terms of exposure of materials to the space environment. The BepiColombo spacecraft cruising toward Mercury will face extreme conditions especially in terms of high-intensity UV radiation and temperature. In this respect, specific materials have been developed to meet the mission requirements. To qualify these materials, there was a need to develop tailor-made testing capabilities. The extensive contribution of Butenko et al. showcases the test performed to assess the suitability of the ceramic white protective coating used on the BepiColombo high gain antenna. Within the same spirit, Tighe et al. describe the results of the highintensity/high-temperature testing performed to qualify the materials and processes for the BepiColombo solar array. At the opposite extreme, ESA's JUICE mission to Jupiter's icy moons will face an extreme radiation environment and low temperature. This environment will, amongst others, induce significant charging of the external materials. Such behavior is investigated in the contribution of Delacourt et al., where several critical materials are tested in vacuum at low temperature and under electron radiation exposure to understand their surface charging behavior.
The last contribution of this special issue, from Snitka et al., deals with the detection of trace amounts of molecular contamination, which is a critical process at all stages of spacecraft assembly and in-flight. The contribution shows how detection of organic molecules can reach sensitivity levels in the ppb range, using a combination of nanomaterials to produce surface enhanced Raman spectroscopy sensors. The outcome of this work shows that besides improvement of molecular species detection at nano-molar level, bio-molecules can also be detected. Apart from having application for cleanliness and contamination control purposes, such technology will also be suitable for environmental control for human space flight application and for detection of organics species on other celestial bodies.

Ugo Lafont and Adrian Tighe, Guest Editors.

Publisher's Note Springer Nature remains neutral with regard to jurisdictional claims in published maps and institutional affiliations. 